\title{
HMD Best Paper Award - Conference Dinner als würdiger Rahmen im 8. Jahr
}

\author{
Matthias Knoll • Sybille Thelen
}

Online publiziert: 5. März 2019

(C) Springer Fachmedien Wiesbaden GmbH, ein Teil von Springer Nature 2019

Der 8. HMD Best Paper Award wurde dieses Jahr erstmals im Rahmen des Conference Dinners der Internationalen Tagung Wirtschaftsinformatik 2019 in Siegen verliehen. Entsprechend groß war die Zuhörerschaft während der Verleihung. Wir bedanken uns herzlich bei den Organisatoren der WI 2019 für diese gerade für die Autorenteams schöne Möglichkeit.

Es hat uns sehr gefreut, dass der HMD Best Paper Award, der in der Community inzwischen sehr gut etabliert ist, dadurch weiter an Sichtbarkeit gewonnen hat. Die Tatsache, dass alle ausgezeichneten Autorenteams unserer Einladung nach Siegen gefolgt sind, unterstreicht den Stellenwert des Awards zusätzlich. Neben der offiziellen Zeremonie bot das Conference Dinner viele Möglichkeiten für regen Austausch und informelle Gespräche aller Beteiligten in angenehmer Atmosphäre.

Für das Jahr 2018 wurden die folgenden Beiträge prämiert:

- Robin Klostermeier, Steffi Haag, Alexander Benlian: Digitale Zwillinge - Eine explorative Fallstudie zur Untersuchung von Geschäftsmodellen, HMD - Praxis der Wirtschaftsinformatik 55 (2018), 320, S. 297-311

Der Beitrag untersucht anhand einer Fallstudie das aktuelle und spannende Thema Digitale Zwillinge. Digitale Zwillinge sind als intelligente digitale Abbilder eines realen Produktes oder Prozesses zu verstehen. Ziel der Fallstudie ist eine erste Untersuchung des digitalen Zwillings aus einer Geschäftsmodellperspektive. Dabei nehmen die vorgestellten Untersuchungsergebnisse Bezug auf neue digitale Geschäftsmodelle und verdeutlichen, dass der digitale Zwilling alle Komponenten

\footnotetext{
M. Knoll ( $\triangle)$

Hochschule Darmstadt, Darmstadt, Deutschland

E-Mail: matthias.knoll@h-da.de

S. Thelen

Springer Vieweg, Wiesbaden, Deutschland
} 
von Geschäftsmodellen beeinflusst. Dabei werden deutliche Unterschiede in der Interpretation der Begrifflichkeit des digitalen Zwillings und dessen Verwendung als Geschäftsmodell sichtbar. Die vergleichende Analyse basiert auf dem Business Model Canvas und nimmt vier unterschiedliche Unternehmenstypen ins Blickfeld.

- Toni Stucki, Sara D’Onofrio, Edy Portmann: Chatbot - Der digitale Helfer im Unternehmen: Praxisbeispiele der Schweizerischen Post, HMD - Praxis der Wirtschaftsinformatik 55 (2018), 322, S. 725-747

Das Autorenteam greift mit dem Beitrag über den Einsatz von Chatbots bei der Schweizerischen Post ein aktuelles und überaus praxisorientiertes Thema auf. Dabei gelingt es, die zentrale Aufgabe der Wirtschaftsinformatik anschaulich und für den Transfer in optimaler Weise zu vermitteln: Ausgehend vom betriebswirtschaftlichen Bedarf bei der Schweizerischen Post geben die Autoren eine übersichtliche technische Einführung in Chatbots, bilden diese IT-Lösung auf den vorliegenden praktischen Anwendungsfall ab und schildern ihre Erfahrungen im Projekt.

- Florian Bartsch, Nils Neidhardt, Markus Nüttgens, Martin Holland, Martin Kompf: Anwendungsszenarien für die Blockchain-Technologie in der Industrie 4.0, HMD - Praxis der Wirtschaftsinformatik 55 (2018), 324, S. 1274-1284

Die Autoren stellen Einsatzszenarien für Blockchain-Anwendungen in Industrie4.0-Umgebungen vor. Als Praxisbeispiel dient hier das durch das Bundesministerium für Wirtschaft und Energie geförderte Projekt ,Secure Additive Manufacturing Platform (SAMPL)“. Primäres Ziel ist es, die insbesondere in der Luftfahrtindustrie komplexe und durch eine Vielzahl anspruchsvoller Vorgaben geprägte Wertschöpfungskette additiv gefertigter Bauteile zuverlässig abzusichern.

In Abb. 1 die angereisten Autoren und Herausgeber sowie Mitarbeiter von Springer Vieweg.

Damit werden nun also bereits im achten Jahr - und im 55. Jahrgang des Journals - die drei besten Beiträge eines Jahrgangs der Zeitschrift „HMD - Praxis der Wirtschaftsinformatik“ mit dem HMD Best Paper Award ausgezeichnet. Die spannende Aufgabe der Auswahl der Gewinner-Beiträge übernimmt das HMD-Herausgebergremium anhand bewährter Kriterien. Betrachtet werden Zielgruppenadressierung, Handlungsorientierung und Nachhaltigkeit, Originalität und Neuigkeitsgehalt, Erkennbarer Beitrag zum Erkenntnisfortschritt, Nachvollziehbarkeit und Überzeugungskraft sowie eine gute Lesbarkeit und Lebendigkeit der Sprache.

Für die prämierten Beiträge, die diese Kriterien in besonderer Weise erfüllen, möchten wir uns mit dem HMD Best Paper Award bei den Autoren bedanken und gleichzeitig unseren Lesern diese Auswahl besonders lesenswerter Texte präsentieren. Dazu erscheinen alle prämierten Beiträge auch in überarbeiteter, meist deutlich erweiterter Fassung als Springer essentials.

Die HMD, seit 2014 bei Springer Vieweg, blickt auf eine sehr lange Geschichte zurück. Unter dem Namen „Handbuch der maschinellen Datenverarbeitung“ wurde sie im Oktober 1964 als Loseblattsammlung erstmals ausgeliefert. Seit 1998 erscheint sie unter dem Titel „HMD - Praxis der Wirtschaftsinformatik“. In den sechs Schwerpunktheften im Jahr tragen sowohl Wissenschaftler als auch Praktiker 


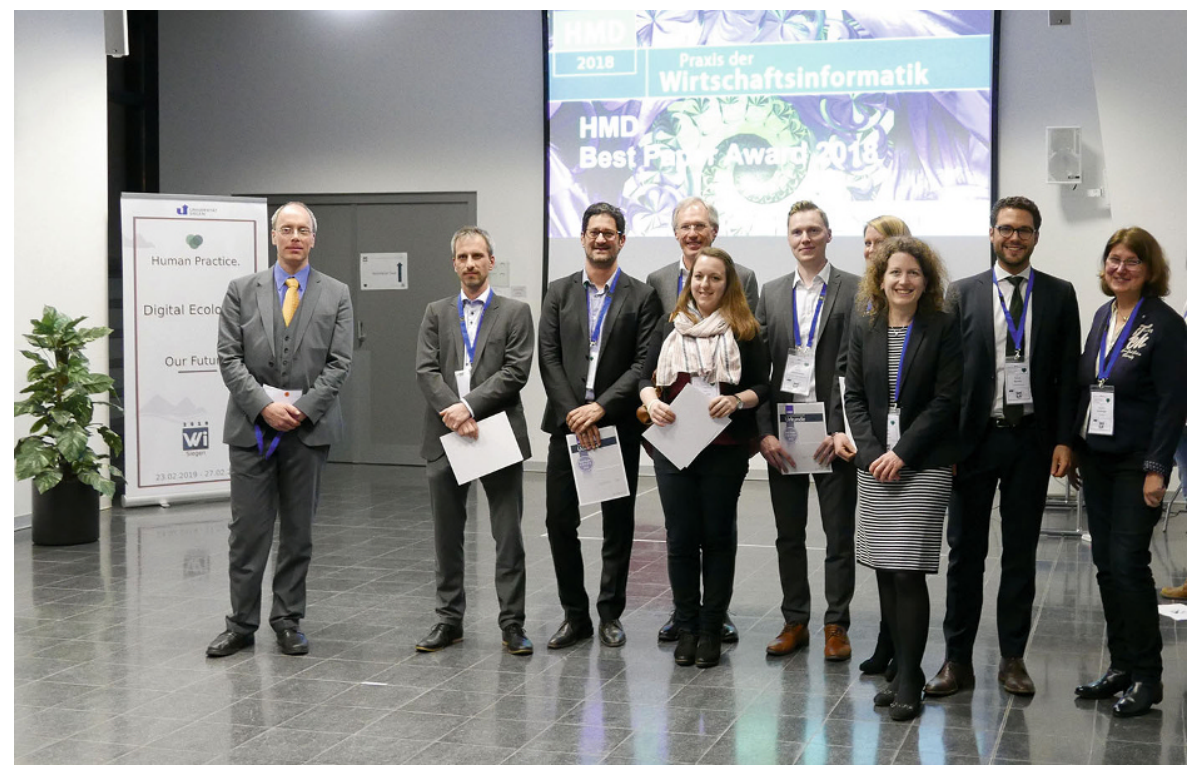

Abb. 1 Verleihung HMD Best Paper Award: (v.l.n.r) M. Knoll, T. Stucki, A. Benlian, S. Meinhardt, S. D’Onofrio, R. Klostermeier, S. Haag, S. Thelen, F. Bartsch, S. Strahringer

durch ihre unterschiedlichen Blickwinkel zu einer differenzierten Betrachtung der jeweiligen Themen bei.

Nicht nur, aber natürlich auch weil mittlerweile neben dem Journal selbst der HMD Best Paper Award zu einer Tradition geworden ist, möchten wir Sie einladen, weiterhin so zahlreiche spannende Beiträge einzureichen und sind uns sicher, dass uns die exzellenten Beiträge nicht ausgehen werden.

Überraschen Sie uns mit neuen Erkenntnissen, Berichten aus der Praxis und spannenden Ideen! Wir freuen uns bereits jetzt auf Ihre Beiträge und die Preisverleihung im März 2020 in Potsdam.

Die Herausgeberinnen und Herausgeber der HMD und der Verlag 